\title{
Design and Analysis of Viscometric Sensor with Embedded Microstructure for Biomedical Applications
}

\author{
Alfredas BRUNIUS*, Christopher RAYAPPAN**, Arvydas PALEVICIUS***, \\ Giedrius JANUSAS $* * * *$, Kęstutis PILKAUSKAS $* * * * *$, Tomas JANUSAS $* * * * * * *$ \\ *Kaunas University of Technology, Studentu 56, Kaunas, Lithuania, E-mail: alfredas.brunius@ktu.lt \\ **Kaunas University of Technology, Studentu 56, Kaunas, Lithuania, E-mail: christopher.rayappan@ktu.edu \\ ***Kaunas University of Technology, Studentu 56, Kaunas, Lithuania, E-mail: arvydas.palevicius@ktu.lt \\ ****Kaunas University of Technology, Studentu 56, Kaunas, Lithuania, E-mail: giedrius.janusas@ktu.lt \\ *****Kaunas University of Technology, Studentu 56, Kaunas, Lithuania, E-mail: kestutis.pilkauskas@ktu.lt \\ ******Kaunas University of Technology, Studentu 56, Kaunas, Lithuania, E-mail: tomas.janusas@ktu.lt \\ crossref http://dx.doi.org/10.5755/j01.mech.24.1.19230
}

\section{Introduction}

Insulin disorder in a human organism is the reason causing diabetes - the disease one of the most spread in the world. The diabetes is distinguished as type 1 and type 2 [1]. Autoimmune attack which destroys beta cells of pancreas results is diabetes of type 1 , while metabolic disorder in the form of high level of glucose in the blood, insulin resistance and relative deficiency of insulin is a is defined as the diabetes of type 2 The Type 2 diabetes, according WHO, covers about $90 \%$ of all the diabetes cases [2]. Therefore, early detection technology convenient and affordable for millions of people suffering from diabetes worldwide is of special need.

Different glucose detection techniques have been developed up to now [3]. Blood glucose (BG) monitoring is the most reliable and highest precision method also called as gold standard method. But the main shortcoming of BG method is the fact that it is invasive method what causes serious discomfort for a patient who needs its frequent application as this is inevitable for glucose level monitoring in case of diabetes diseases [4]. The situation stimulates the searches for more comfortable - non-invasive methods. Therefore, numerous research efforts aimed at the development of non-invasive methods are being made [5, 6]. Techniques of glucose detection in other human serums such as saliva, sweat, tears, urine or even exhaled breath (gas) have been developed or currently are under development. They still lack confidence of the obtained results as great number of facts makes impact on biochemical composition of the mentioned serums and response time constant is not yet sufficient if compared to BG method. Nevertheless, the latest achievements in biotechnologies, micro and nano technologies allow promising expectations for convenient non-invasive glucose level monitoring techniques development [7].

Development of glucose level monitoring techniques evolves by two major paths - electrochemical sensing technology and physical properties changing of a sensing fluid due to affinity binding principles, e.g. in solution of dextran concavalin A (con A) [2, 5]. An electromechanical sensor consists of the basic part bio receptor - in the most cases it is glucose oxidace (GOx) enzyme which in reaction with glucose generates electron transport signal that is converted into measurable electric signal by second basic part of the sensor - transducer. Nevertheless, electrochemical sensors still feature drawbacks such as accumulation of reaction products, non-sufficient stability and response time. Typical technologies developed according the second path can be represented by viscometric sensors [8]. The basic functional part of the sensor is micro cantilever immersed into a fluid the viscosity of which is changed due to binding of glucose to con A. The increase of glucose level results in decrease of the solution what in turn results in the change of response characteristics of the micro cantilever when it is excited by periodical or discrete signal. Vibration characteristics of the cantilever are detected using optical lever system with the help of light beam reflected from its surface or using indirect capacity measurement method [9]. Nevertheless, the applied principles of vibration measurements are inconvenient for practical (clinical) application cases.

In order to make measurement system more convenient, increase measurement accuracy and get the sensor that can be manufactured applying cheap manufacture processes a novel structure glucose sensor is proposed in the paper. The main focus is made on the design of viscoelastic cantilever type sensors with the embedded piezo-active functional element to drive micro cantilever in order to detect concentration of glucose in the physiological liquid. Analytical, numerical and experimental methods for design and analysis of viscoelastic sensor are presented.

\section{Design}

Micro cantilever design as shown in Fig.1, is particular in that it has the electrical contacts which are fixed onto the novel nano composite piezoelectric polymer. Nano composite piezoelectric polymer was developed by mixing PZT powder with $20 \%$ solution of polyvinyl butyral in benzyl alcohol. For the numerical and theoretical analysis nano composite material with $80 \%$ concentration of PZT powder was selected. It ensures the higher sensitivity at lower resonant frequency as this composite material has lower stiffness than PZT ceramics. This piezoelectric material was screen printed on an $\mathrm{Al}$ coated polyvinyl chloride (Al-PVC) using 325 mesh stainless steel screen, dried for $30 \mathrm{~min}$ at $100^{\circ} \mathrm{C}$ and poled using electrical field of $5 \mathrm{kV}$. Moreover, periodic microstructure can be embedded in order to increase the functionality of the electro-optic active element [10].

The schematic representation of the principle design of the proposed sensing element for glucose detection 
is shown in Fig. 1. Its main structural unit consist from AlPVC beam as the base and PZT nano composite layer at the end covered by $\mathrm{Al}$ (electrical contact). When applying voltage onto the contacts, the material due to its piezoelectric properties excites the end of cantilever and in case of the glucose concentration change in the liquid where it is immersed the feedback in the form of frequency shift of micro cantilever response is obtained. The effective usage of such type sensing element requires to perform analytical, numerical and experimental investigations. Therefore, the analytical equation of motion and finite element analysis are presented in the next chapter.

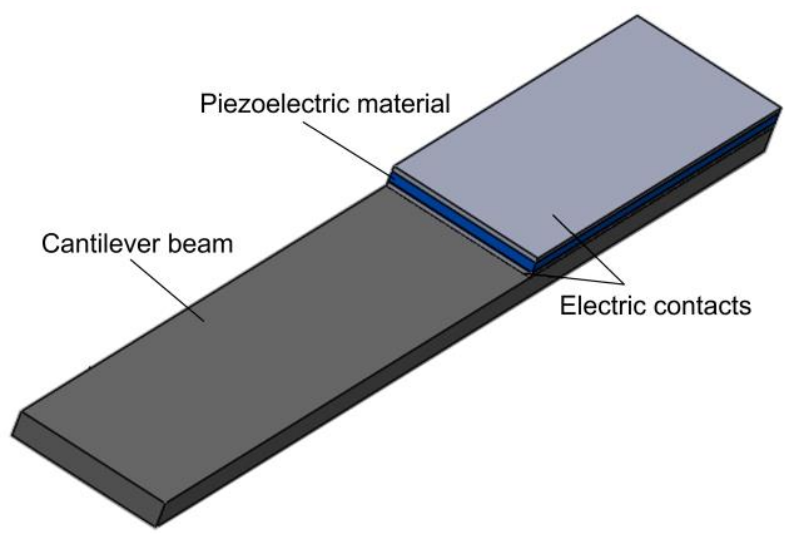

Fig. 1 Cantilever beam design with the electrical elements

\section{Mathematical modelling}

Mathematical modelling as a method of structural analysis, is particularly suitable for computer automated analysis of complex structural models. In the analysed structure, the cantilever beam is permanently fixed at one end and the other end is free to deflect under the effect of gravity.

The piezoelectric and other functioning together layers can be represented as single two dimensional horizontally oriented finite beam element with concentrated mass as shown in Fig. 2.

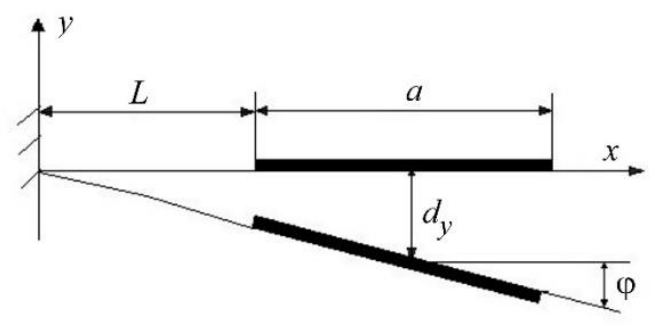

Fig. 2 Schematic representation of the cantilever with its essential geometrical parameters [9]

They are approximated as a plate of mass $m$ and length $a$; attached to elastic beam which under the effect of electrical torque deflects by $d_{y}$ from the centre of mass along the $y$-axis and deviates by the angle $\theta$ in plane $x y$ [9].

In elastic metal beam which serves as the base element of the sensor under the effect of transversal loads bending, twisting and axial deformations can be observed. Transversal and rotational displacements are the characteristics of bending deformation. Therefore mobility at a node of a beam element are expressed as a transversal displacement and rotation. Stiffness matrix is formed for each node using the same sign reference system as follows:

- Counter-clockwise direction is an assumed to be positive for Moments and rotations.

- Positive direction of Y axis is assumed as positive for Displacements and Forces

Taking into account that the plate has 2 DOF its displacement vector is $u=\left[d_{y}, \varphi\right]^{T}$, where $L$ is length of the beam not covered by the piezoelectric material plate.

The cantilever mass is negligible insignificant if compared to the plate mass $m$, therefore it is modelled as ideal elastic element. Form the theory of elastic beams elasticity force is:

$$
F_{e l}=12\left[d_{y}-\frac{1}{2} \alpha \varphi\right]-6 \varphi
$$

and torque:

$$
T_{e l}=-6\left[d_{y}-\frac{1}{2} \alpha \varphi\right]+4 \varphi,
$$

where: $\left(d_{y}-\frac{1}{2} \alpha \varphi\right)$ is the displacement of free end of cantilever beam. Spring vector of Elasticity force is:

$$
R_{e l}=\left[F_{e l}, T_{e l}\right]^{T}
$$

Using beam theory, the shear force of the beam is calculated as:

$$
\hat{F}_{e l}=-E I \frac{\partial^{3} v}{\partial x^{3}}=\frac{E I}{L^{3}}\left[12 d_{y}-6 L\left(1+\frac{\alpha}{L}\right)\right] \varphi .
$$

Similarly, the bending moment is obtained using:

$$
\hat{T}_{e l}=E I \frac{d^{2} v}{d x^{2}}=\frac{E I}{L^{3}}\left[-6 L d_{y}+\left(4+\frac{3 \alpha}{L}\right) L^{2}\right] \varphi,
$$

where $v$ is transverse displacement function:

$$
\begin{aligned}
& v=\left[-\frac{2}{L^{3}} d_{y}+\frac{1}{L^{2}}\left(1+\frac{\alpha}{L}\right) \varphi\right] x^{3}+ \\
& +\left[\frac{3}{L^{2}} d_{y}-\frac{1}{L^{2}}\left(1+\frac{3}{2 L}\right) \varphi\right] x^{2} .
\end{aligned}
$$

Writing above in matrix form we get:

$$
\left[\begin{array}{l}
\hat{F}_{e l} \\
\hat{T}_{e l}
\end{array}\right]=\frac{E I}{L^{3}}\left[\begin{array}{cc}
12 & -6 L\left(1+\frac{\alpha}{L}\right) \\
-6 L & \left(4+\frac{3 \alpha}{L}\right) L^{2}
\end{array}\right]\left[\begin{array}{l}
d_{y} \\
\varphi
\end{array}\right],
$$


where: $K=k\left[\begin{array}{cc}12 & -6 L\left(1+\frac{\alpha}{L}\right) \\ -6 L & \left(4+\frac{3 \alpha}{L}\right) L^{2}\end{array}\right]$ is stiffness matrix of the beam and $k=\frac{E I}{L^{3}}$. Here $E$ is Young's modulus of the beam material. We know that $R_{\text {elect }}=K u$, where $K$ is stiffness matrix of the cantilever. Here $F_{f}$ and torque $M_{f}$ are the forces acting on the plate due to fluid - plate interaction and can be obtained from the solution of adequately formulated problem in fluid dynamics. Due to viscous and inertia properties of the fluid they can be considered as dependant on motion parameters (velocity and acceleration) of the plate [9]. Applying vector notation, the fluid force can be expressed as follows:

$$
R_{F}=\left[F_{f}, T_{f}\right]^{T}
$$

But the force is:

$$
R_{F}=T_{f} \ddot{u}+D_{f} \dot{u},
$$

where: $M_{f}$ and $D_{f}$ is the mass and fluid damping matrixes accordingly. Then oscillatory motion of the plate can be expressed:

$$
\begin{aligned}
& M \ddot{d}_{y}+F_{e l}+F_{f}=0, \\
& \frac{1}{2} M \alpha^{2} \ddot{\varphi}+T_{e l}=F_{e l}\left(\frac{1}{2} \alpha\right)-T_{f}-T_{\text {elect }} .
\end{aligned}
$$

According to Newton's second law, the equation of motion of the damped system damping is given as:

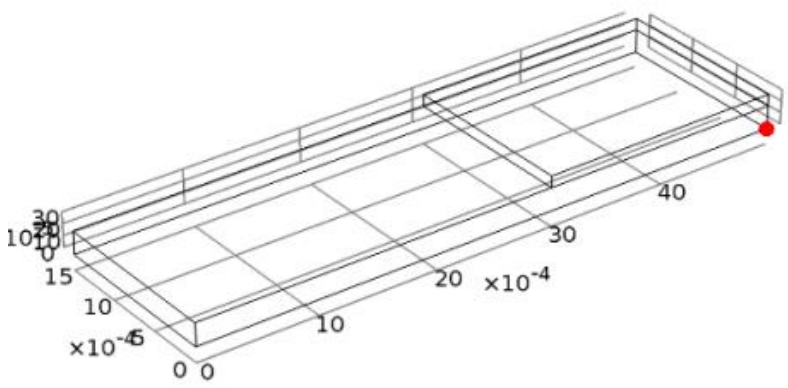

a

$$
M \ddot{u}+C \ddot{u}+K u=F .
$$

For our case, the acceleration is in direction and the damping, as well as spring force, is zero. In matrix form, it is given as:

$$
\left(M+M_{f}\right) \ddot{u}+D_{f} \dot{u}+K u=R_{\text {elect }},
$$

where the mass matrix, stiffness matrix, and electric force vector are as follows [9]:

$$
M=\left[\begin{array}{cc}
m & 0 \\
0 & \frac{1}{12} m(\alpha / L)^{2}
\end{array}\right],
$$

$$
K=k\left[\begin{array}{cc}
12 & -6 L\left(1+\frac{\alpha}{L}\right) \\
-6 L\left(1+\frac{\alpha}{L}\right) & 4 L^{2}+6 a L+27 a^{2}
\end{array}\right],
$$

$$
R_{\text {elect }}=\left[\begin{array}{c}
0 \\
\frac{T_{\text {elect }}}{L}
\end{array}\right] .
$$

\section{Numerical analysis}

In order to know the modes of vibration of micro cantilever FEM model is build (Fig. 3). The model provides investigation of transient processes as well (Fig. 4-Fig. 5). The modelling of vibrating micro cantilever is done with the help of COSMOL MULTIPHYSICS. The results of calculations are useful for design or manufacture of the micro cantilever. FEM mesh analysis model and simulation parameters are presented in Fig. 3(b) and Table 1.

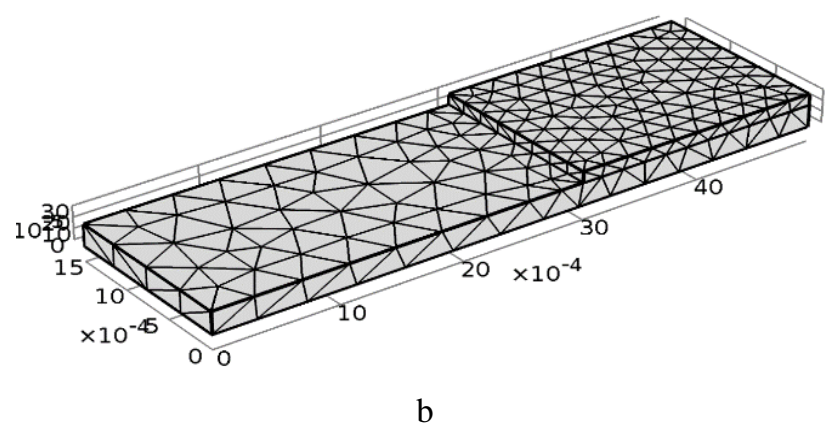

Fig. 3 (a) Transient analysis point location, (b) Meshed analysis model

Table 1

Microcantilever parametric table

\begin{tabular}{|l|l|l|}
\hline \multicolumn{1}{|c|}{ Name } & \multicolumn{1}{|c|}{ Expressions } & \multicolumn{1}{c|}{ Value } \\
\hline$T_{\max }$ & $50 /$ freq & 0.010960 \\
\hline$T_{\text {step }}$ & $T_{\max } * 1 \mathrm{e}-2$ & $1.0960 \mathrm{E}-4$ \\
\hline Freq & $4562 \mathrm{~Hz}$ & $4562.0 \mathrm{~Hz}$ \\
\hline Amp & $40 \mathrm{~V}$ & $40 \mathrm{~V}$ \\
\hline
\end{tabular}




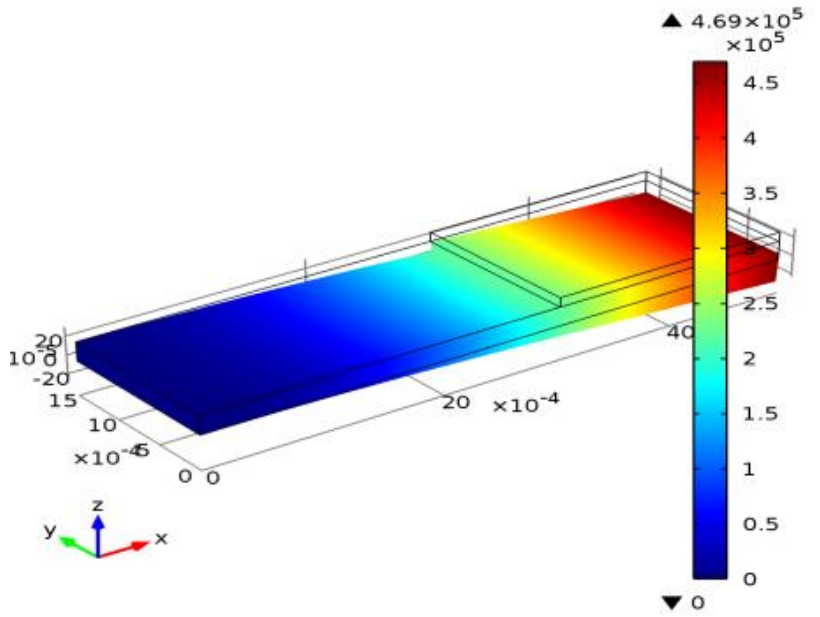

a

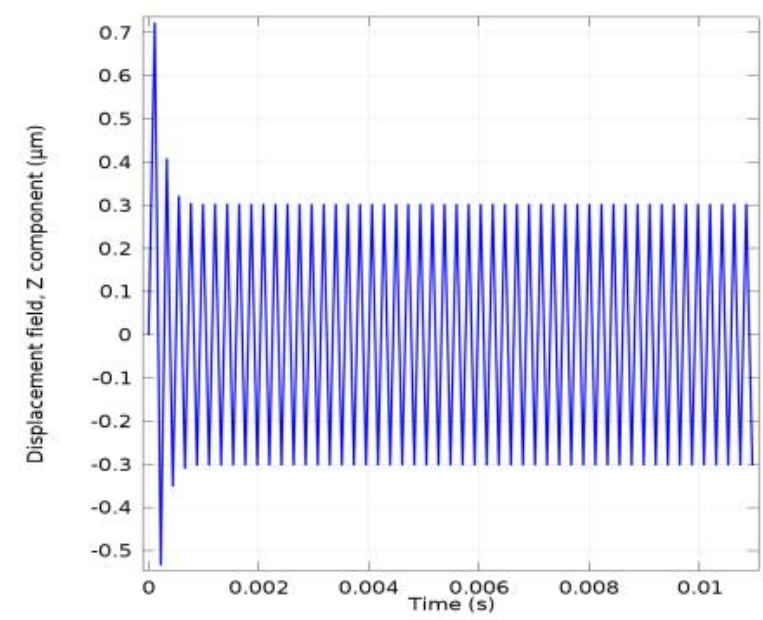

$\mathrm{b}$

Fig. 4 (a) Transient analysis model without damping, (b) Graph for Transient analysis model without damping

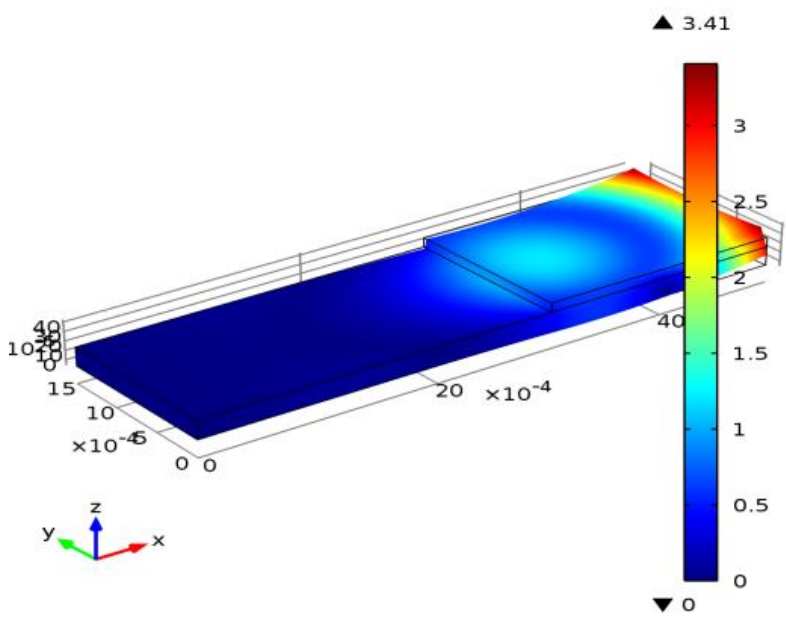

a

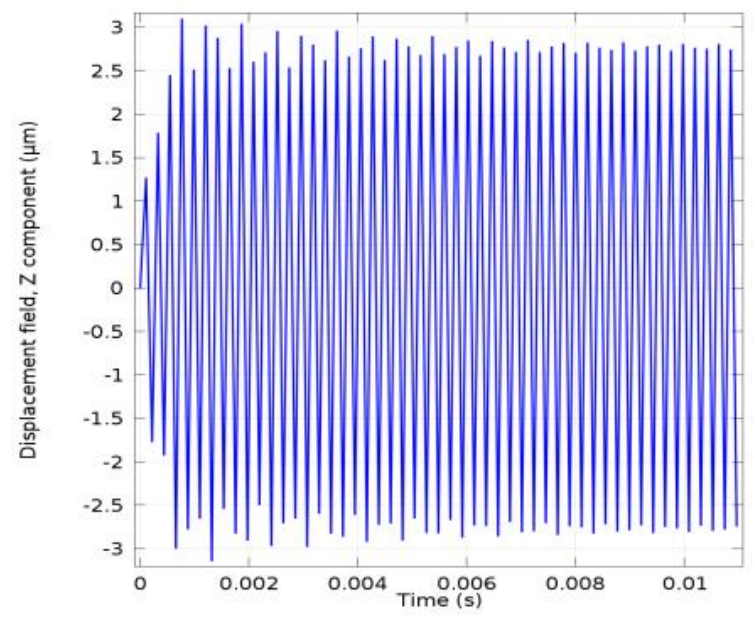

$\mathrm{b}$

Fig. 5 (a) Transient analysis model with damping, (b) Graph for Transient analysis model with damping

The results of simulation were useful for determining the main geometric parameters of viscoelastic micro sensor and the main optimal working regimes of the cantilever. It was shown that the theoretically determined frequency of the proposed cantilever is $1.6 \mathrm{kHz}$, when cantilevers dimensions are: length $-50 * 10^{-4} \mathrm{~m}$, width $-15^{*} 10^{-4} \mathrm{~m}$, thickness $-2 * 10^{-4} \mathrm{~m}$. Dimensions of piezoelectric element are: length $-20 * 10^{-4} \mathrm{~m}$, width $-15^{*} 10^{-4} \mathrm{~m}$, thickness $1 * 10^{-4} \mathrm{~m}$.

\section{Experimental results}

Model of the cantilever Manufactured from polyvinyl chloride (PVC) material is used in this experiment. One side of the cantilever is anchored with help of unmovably fixing bracket and the other side is suspended to the gravity. The plate of piezoelectric composite material with electrical contacts is attached to the open end of cantilever. The contacts are connected to the vibration parameter measurement system Pico Scope the data from which is transferred to computer, processed by the Pico Scope software and outputted in the form of graphs (variation of amplitude and time are presented in the graph) (Fig. 6). The bump-test was made three times and the best results are shown in Fig. 7.

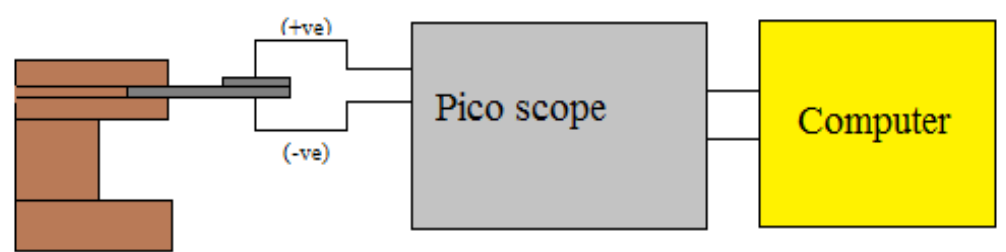

Fig. 6 Experimental setup of cantilever vibration analysis 


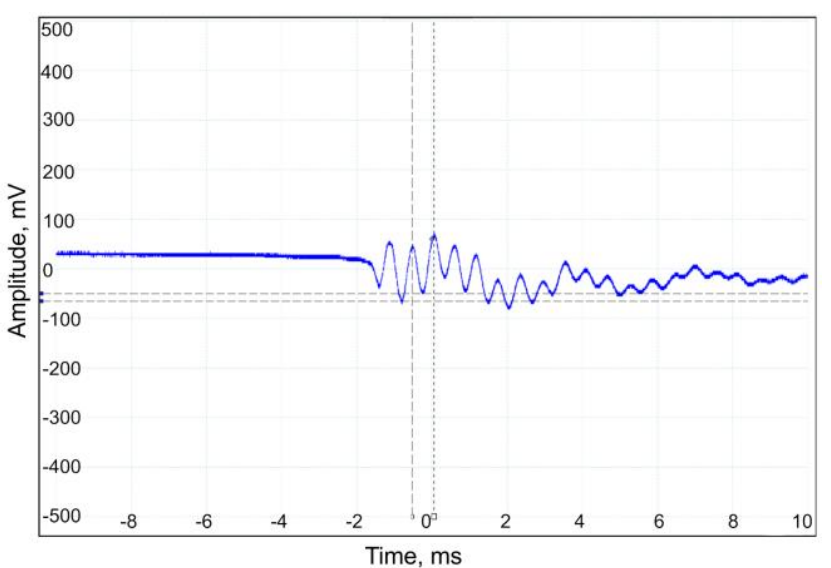

a

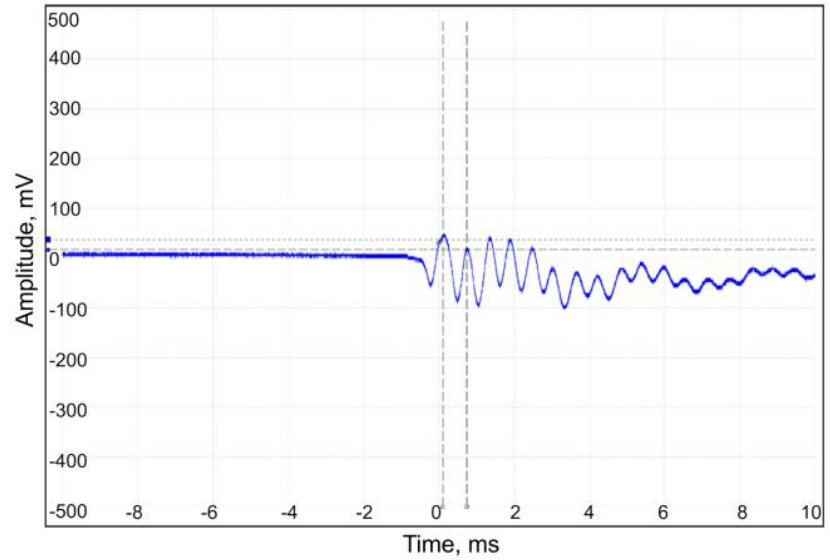

b

Fig. 7 Bump-test results: a-test 1 ; b- test 2

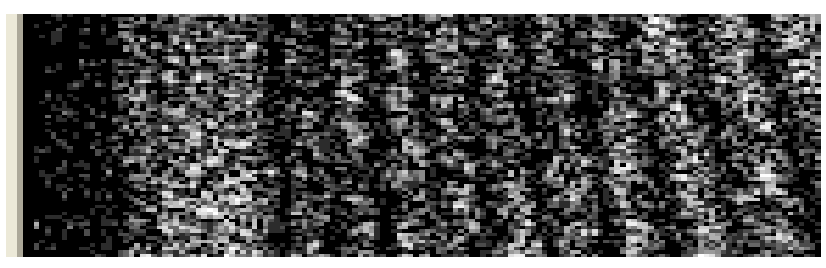

a

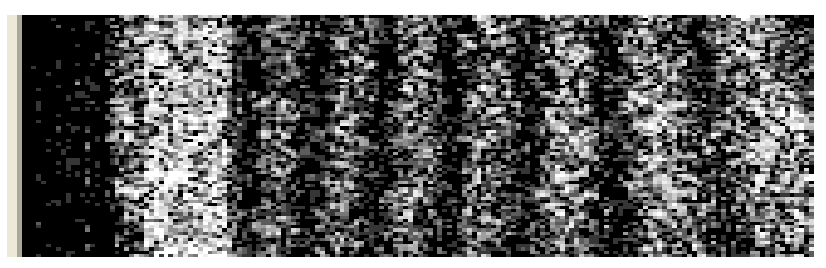

$\mathrm{b}$

Fig. 8 Holographic interferogram of vibrating micro cantilever in liquid: a-liquid without glucose; b-liquid with glucose

The frequency response interval is $1.5 \mathrm{kHz}$ to $1.7 \mathrm{kHz}$. The theoretical value is comparatively equal to the experimental frequency value.

To verify created FEM model and for visualization of mode vibration of cantilever the method of holographic interferometry was used [11]. The holographic interferograms of working viscometric sensor in the liquid without glucose and with glucose are presented in Fig. 8. They show that amplitude of cantilever vibrating in liquid with glucose and excited at $1.6 \mathrm{kHz}$ was decreased about $30 \%$ in comparison with vibrations in liquid without glucose. The described phenomena allow to create noninvasive sensor for glucose level detection in various physiological liquids.

\section{Conclusions}

The cantilever activated by electrical elements instead of using magnetic excitation was designed and analyzed. As in the known models some difficulties to place magnet in the sensor exist, the magnet was replaced by thin piezoelectrical element used to excite the micro cantilever; together the possibility to variate easily the size of cantilever appeared.

FEM analysis of vibration forms of the cantilever enabled to determine appropriate operation frequency which in the case of analysis was equal to $1.6 \mathrm{kHz}$.

The obtained results of experimental analysis are in agreement with the results of FEM analysis what proves adequacy of FEM model. The main suitable experimentally determined operation frequency be used in sensors for glucose detection is in the range between $1.5 \mathrm{kHz}$ to $1.7 \mathrm{kHz}$.

\section{Acknowledgment}

This research was funded by a grants (No. MIP081/2015 and S-MIP-17-102) from the Research Council of Lithuania.

\section{References}

1. Janghorbani, M.; Van Dam, R.M.; Willett, W.C.; Hu, F.B. 2007. Systematic Review of Type 1 and Type 2 Diabetes Mellitus and Risk of Fracture, American Journal of Epidemiology 166(5): 495-505. https://doi.org/10.1093/aje/kwm106.

2. Casagrande, S.S.; Cowie, C.C. 2012. Health insurance coverage among people with and without diabetes in the U.S. Adult Population, Diabetes Care 35(11): 22432249. http://dx.doi.org/10.2337/dc12-0257.

3. Galant, A.L.; Kaufman, R.C.; Wilson, J.D. 2015. Glucose: Detection and analysis, Food Chemistry 188: 149-160. http://dx.doi.org/10.1016/j.foodchem.2015.04.071.

4. Vashist, S.K. 2013. Continuous Glucose Monitoring Systems: A Review, Diagnostics 3(4), 385-412. http://dx.doi.org/10.3390/diagnostics3040385.

5. Seo, D.; Paek, S.H.; Oh, S.; Seo, S.; Paek, S.H. 2016. A Human Serum-Based Enzyme-Free Continuous Glucose Monitoring Technique Using a Needle-Type BioLayer Interference Sensor, Sensors 16(10): 1-7. http://dx.doi.org/10.3390/s16101581.

6. Kottmann, J.; Rey, J.M.; Sigrist, M.W. 2016 Mid-Infrared Photoacoustic Detection of Glucose in Human Skin: Towards Non-Invasive Diagnostics, Sensors 16(10): 1-14. http://dx.doi.org/10.3390/s16101663.

7. Zhang, W.; Du, Y.; Wang, M.L. 2015. Noninvasive glucose monitoring using saliva nano-biosensor, Sensing and Bio-Sensing Research 4: 23-29. https://doi.org/10.1016/j.sbsr.2015.02.002.

8. Huang, X.; Li, S.; Davis, E.; Leduc, C.; Ravussin, Y.; Cai, H.; Song, B.; Li, D.; Accili, D.; Leibel, R.; 
Wang, Q.; Lin, Q. 2013. A MEMS differential viscometric sensor for affinity glucose detection in continuous glucose monitoring, Journal of Micromechanics and Microengineering 23(5): 1-10.

https://doi.org/10.1088/0960-1317/23/5/055020.

9. Zhao, Y.; Li, S.; Davidson, A.; Yang, B.; Wang, Q.; Lin, Q. 2007. A MEMS viscometric sensor for continuous glucose monitoring, Journal of Micromechanics and Microengineering 17(12): 2528-2537. https://doi.org/10.1088/0960-1317/17/12/020.

10. Janusas, G.; Palevicius, A.; Ostasevicius, V.; Bansevicius, R.; Busilas, A. 2007. Development and experimental analysis of piezoelectric optical scanner with implemented periodical microstructure, Journal of vibroengineering 9(3): 10-14.

https://doi.org/10.1117/12.776762.

11. Ostasevicius, V.; Palevicius, A.; Daugela, A.; Ragulskis, M.; Palevicius, R. 2004. Holographic Imaging technique for characterization of MEMS switch dynamics, Smart structures and materials 2004: smart electronics, mems, biomems and nanotechnology 5389: 73-84. http://dx.doi.org/10.1117/12.540183.
A. Brunius, Ch. Rayappan, A. Palevicius, G. Janusas, K. Pilkauskas, T. Janusas

\section{DESIGN AND ANALYSIS OF VISCOMETRIC SENSOR WITH EMBEDDED MICROSTRUCTURE FOR BIOMEDICAL APPLICATIONS}

\section{S u m m a r y}

The aim of this paper is to propose a novel structure glucose sensor which makes glucose level measurement system more convenient, increases measurement accuracy and gets the sensor that can be manufactured applying cheap manufacture processes. The main focus is made on the design of viscoelastic cantilever type sensors with the embedded piezo-active functional element to drive micro cantilever in order to detect concentration of glucose in the physiological liquid. Analytical, numerical and experimental methods for design and analysis of viscoelastic sensor are presented.

Keywords: biomechanical sensors; cantilever; mathematical modelling. 